\title{
The mixing layer at high Reynolds number: large-structure dynamics and entrainment
}

\author{
By PAUL E. DIMOTAKIS \\ AND GARRY L. BROWN† \\ Graduate Aeronautical Laboratories, California \\ Institute of Technology, Pasadena
}

(Received 7 November 1975 and in revised form 19 March 1976)

\begin{abstract}
A turbulent mixing layer in a water channel was observed at Reynolds numbers up to $3 \times 10^{6}$. Flow visualization with dyes revealed (once more) large coherent structures and showed their role in the entrainment process; observation of the reaction of a base and an acid indicator injected on the two sides of the layer, respectively, gave some indication of where molecular mixing occurs. Autocorrelations of streamwise velocity fluctuations, using a laser-Doppler velocimeter (LDV) revealed a fundamental periodicity associated with the large structures. The surprisingly long correlation times suggest time scales much longer than had been supposed; it is argued that the mixing-layer dynamics at any point are coupled to the large structure further downstream, and some possible consequences regarding the effects of initial conditions and of the influence of apparatus geometry are discussed.
\end{abstract}

\section{Introduction}

It is becoming increasingly evident that the classical picture of turbulence, as a state of flow in which the properties of the various dynamic variables can only be determined in a stochastic sense, is not quite complete. Experimental results in the last few years indicate that within the obvious randomness of turbulence there exist flow patterns and large-scale structures that appear to be dominant in determining the overall characteristics of such flows. A notable example is the discovery that the turbulent mixing layer is inhabited by a more or less organized large structure (Brown \& Roshko 1971).

Large-scale vortices and instabilities at very high Reynolds number had been found in other flows. Yet the apparent quasi-regularity that is manifest in the photographs and high-speed motion pictures of Brown \& Roshko (1971, 1974) was not expected. It was unexpected because such well-defined structure is far from random and appears inconsistent with the classical picture of turbulence. Even though such apparent inconsistencies are probably adequately explained, in terms of the irregularity and jitter in the formation, amalgamation and spacing of these large structures (Brown \& Roshko 1974), important questions remain to be answered. It becomes important, for example, to establish whether

$\dagger$ Permanent address: University of Adelaide, Australia. 
these large structures persist at even higher Reynolds numbers or whether they are a curious transition from the well-defined periodicity of the laminar instability region (Sato 1956) to a state of complete randomness at infinite Reynolds number. In addition, the question of persistence of the mechanism of vortex pairing, well documented at relatively low Reynolds number (Winant \& Browand 1974), arises, especially since this mechanism is not so apparent in the photographs and motion pictures of Brown \& Roshko, taken at much higher Reynolds numbers.

With these questions in mind, the experiment described here was undertaken. It was decided to use the GALCIT $50 \times 50 \mathrm{~cm}$ Free Surface Water Tunnel, which has a test section $2.4 \mathrm{~m}$ ( $8 \mathrm{ft}$ ) long and in which a test-section velocity of $7.6 \mathrm{~m} / \mathrm{s}$ can be reached. To generate the shear layer, an insert was designed for the test section, yielding a velocity ratio between the two streams of $5: 1$.

Water offers several attractive features as the flowing medium. The most important of these, for our purposes, was the high Reynolds number per unit length and velocity. In addition, and as a consequence of the relatively low velocities required, flow visualization by dye injection is particularly simple. Lastly, it was possible to take advantage of the high accuracy and high spatial and temporal resolution of a newly developed single-particle laserDoppler velocimeter, water being an ideal medium for this measurement technique.

By injecting dye for flow visualization, we were able to observe and photograph the large structure and follow the process of entrainment by direct observation and motion pictures. By replacing the dye by an acid containing a $\mathrm{pH}$ indicator on one side and by a base on the other side of the layer, we obtained photographs as well as a motion picture in which the chemical reaction between the two, as they were entrained and mixed in the layer, was qualitatively marked by the change in colour of the indicator.

The flow-visualization evidence for the existence of the large structure at a high Reynolds number $\left(3 \times 10^{6}\right)$ is unmistakable and its dominant role in the entrainment process is manifest.

The autocorrelation function of the streamwise velocity fluctuations revealed a fundamental periodicity which was found to be consistent with the expected similarity scaling. Other features of the data, however, cannot be explained quite so simply and it now appears that similarity in the broad sense does not account for much of the observed behaviour. More complicated processes which cannot be explained in terms of local phenomena seem to govern the dynamics of the flow. Mechanisms for coupling the whole shear layer are required and suggest that such factors as initial conditions may always be important.

These experiments are the first part of a larger investigation and perhaps raise more questions than they can answer. Nevertheless, we feel that the results presented here are of sufficient interest in themselves and important in that they do raise these questions. 


\section{Apparatus and resulting flow fluid}

The high Reynolds numbers which may be achieved in the $50 \times 50 \mathrm{~cm}$ GALCIT Free Surface Water Tunnel made this facility a logical choice for the experiment. This tunnel is capable of a maximum velocity, without a model in the $2.4 \mathrm{~m}$ working section, of $7 \cdot 6 \mathrm{~m} / \mathrm{s}$. It was decided to modify this facility in such a way as to generate a well-defined, two-dimensional shear layer with a high aspect ratio. Unfortunately, to obtain a shear layer with a large velocity ratio in two separate streams within the test section of a conventional wind or water tunnel is by no means straightforward. This is especially true if it is not feasible to divide the flow into two parts within the tunnel contraction. In several other attempts to establish two streams of different velocity in a tunnel test section by dividing the flow and placing flow resistance on the low-speed side, there appear to have been difficulties with flow separation and flow non-uniformity as the velocity ratio became large. Having a velocity of zero on the low-speed side may appear to avoid these problems but, in the GALCIT Water Tunnel at least, to satisfy the entrainment requirements while maintaining a low-turbulence high-quality flow would render this choice an even more difficult problem. Consequently, we decided to design an insert that would produce a shear layer with a non-zero $U_{2}$ within the water-tunnel test section, taking special precautions to minimize the possibility of separation. The outcome is shown in figure 1 and the manner in which it is placed in the working section is shown in figure 2. The resulting apparatus proved to be very satisfactory and the basis for the design of the insert possibly merits a brief description. In order to avoid separation at the leading edge of the flow divider, it is desirable to design the two flow paths to have exactly the same pressure drop for a given inlet velocity. For an area contraction ratio of $A_{\mathrm{in}} / A_{\text {out }}=C_{A}$ on the high-speed side and a corresponding expansion of $1 / C_{A}$ on the low-speed side, matched inlet and exit pressures and uniform flow at the two inlets ensure that the velocity ratio $r \equiv U_{1} / U_{2}$ is equal to $C_{A}^{2}$. The flow resistance on the low-speed side must match the Bernoulli pressure drop on the high-speed side. The flow must also be redirected within the expansion to avoid separation. The two perforated plates shown in figure 1 (50\% open) were accordingly placed where separation might otherwise have occurred. The plates were curved (rolled as segments of a circle) so as to be approximately perpendicular to the streamlines of an inviscid flow through the expansion. Adjustment was provided by allowing movement of the lower edge of the plate upstream and the upper edge downstream or vice versa so that the flow could be directed more towards the lower or upper boundary. Fine control and adjustment of the pressure drop were achieved by placing an identical perforated sheet on top of the first. Sliding one vertically relative to the other changed the percentage open area. This, of course, had some effect on the flow direction after the plate, which had to be offset, as required, by tilting the plate. Finally, the resulting turbulence on the low-speed side was reduced in scale and amplitude and the flow made even more uniform by the 40 mesh, $50 \%$ open screen placed at the exit of the expansion. The percentage open areas (solidities) of the plates and the screen as well as their locations were chosen to 


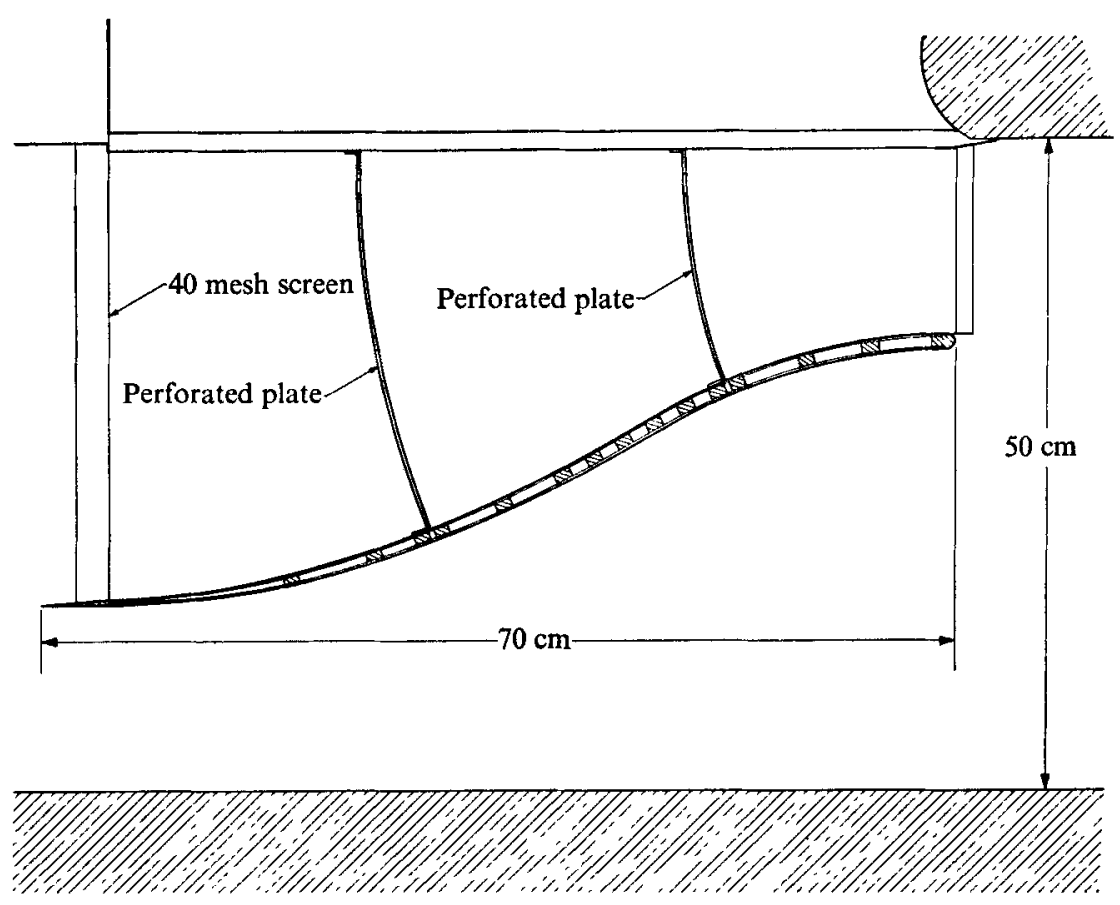

Figure 1. Insert for the shear layer with velocity ratio 5: 1.

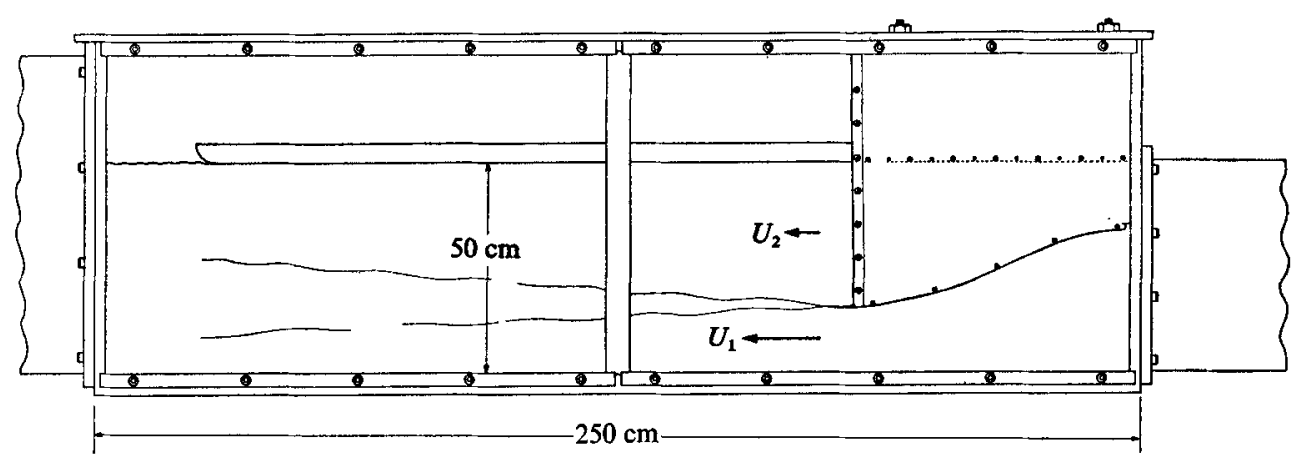

FIGURE 2. Test section with insert and top installed.

provide the requisite total pressure coefficient. Handbook data were used to compute the individual pressure drops. Perforated plates were chosen to provide the flow resistance because the pressure drop across them is very nearly Reynolds number independent and because they have sufficient thickness to provide in-plane forces that tend to give an exit flow velocity that is perpendicular to the surface of the plate (hence the curved shape). In addition to this insert, a lucite top $150 \mathrm{~cm}$ long was installed in the remainder of the test section to remove free-surface effects of various kinds (see figure 2).

Just downstream of the insert, the flow on the low-velocity side was found to be uniform to within $1 \%$ of the velocity of the faster stream over the full $35 \mathrm{~cm}$ 


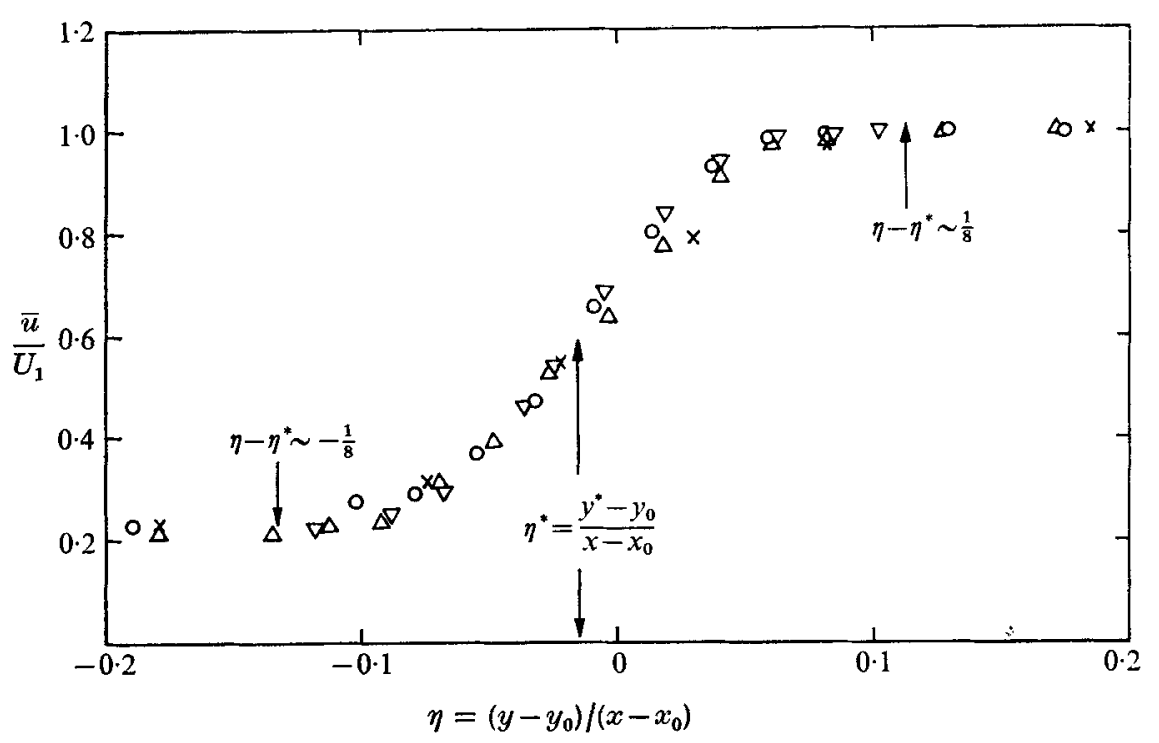

Fiaure 3. Similarity mean velocity profiles. $x, x=15.24 \mathrm{~cm} ; O, x=30.48 \mathrm{~cm} ; \triangle$, $x=60.96 \mathrm{~cm} ; \nabla, x=91.44 \mathrm{~cm} . x_{0}=3.05 \mathrm{~cm}$. Free-stream velocity on high-spe日d side $U_{1}=165 \mathrm{~cm} / \mathrm{s}$.

width of the flow. The measured velocity ratio $r=U_{2} / U_{1}$ was 0.21 with $U_{1}=165$ $\mathrm{cm} / \mathrm{s}$. At the first measuring station, $15 \mathrm{~cm}$ downstream from the end of the splitter plate, the root-mean-square fluctuation of the velocity measurements in the free stream on the high-speed side was less than $0.5 \%$ of $U_{1}$ (no attempt was made to distinguish low frequency velocity fluctuations, turbulence and electronic noise: this is then an upper bound for the turbulence level). On the low-speed side it was less than $2 \%$ of $U_{2}$, which, since $U_{2}$ is much less than $U_{1}$, is also very small compared with the relevant velocity $U_{1}-U_{2}$. In general, the whole flow was very steady (less than $1 \%$ long-term variation in free-stream velocity) over many hours of operation, except for some small low frequency fluctuations in free-stream velocity, observed at the measuring station furthest downstream, probably owing to surface waves downstream of the $150 \mathrm{~cm}$ lucite top.

At a free-stream velocity on the high-speed side of $165 \mathrm{~cm} / \mathrm{s}$, the boundary layer was found to be transitional (occasional turbulent spots), with a momentum thickness of the order of $0.4 \mathrm{~mm}$. The maximum slope thickness, $12 \mathrm{~mm}$ downstream of the splitter plate's trailing edge, was $2 \mathrm{~mm}$. The mean velocity profiles at a high-speed free-stream velocity of $165 \mathrm{~cm} / \mathrm{s}$, at which most of the data were taken, are plotted in similarity co-ordinates in figure 3. They represent measurements $15 \mathrm{~cm}, 30 \mathrm{~cm}, 60 \mathrm{~cm}$ and $90 \mathrm{~cm}$ downstream of the splitter plate. Each point is the time-averaged velocity computed from several records consisting of 1024 discrete measurements, in a manner described in $\$ 4$. No quantitative velocity data were recorded any further downstream in order to restrict the data to an aspect ratio for the large structure (ratio of span to vorticity thickness) as large as possible. From the flow-visualization data, however, it was clear that, at least qualitatively, the same basic two-dimensional large structure persisted 
to the end of the working section, i.e. for an additional distance about twice that of the furthest measuring station. See figure 4 (plate 1).

We were surprised to find the scatter in the mean profile data to be as large as it was. For those points, particularly near the dividing streamline, where several records of 1024 measurements were taken, variations of as much as 5-10\% were found between estimates of the mean velocity obtained from each record. It now seems likely that the averaging intervals during which the 1024 velocity samples were recorded may not have been long enough. This becomes a more definite possibility in view of the surprisingly long time scales that were found to characterize the flow dynamics.

The mean velocity profile does not seem to have attained the self-similar shape by $x=15 \mathrm{~cm}$. The vorticity (maximum slope) thickness for stations further downstream was estimated to satisfy

$$
0 \cdot 10 \lesssim \delta_{\omega} /\left(x-x_{0}\right) \lesssim 0 \cdot 13
$$

This is consistent with the range of values of the data collected by Brown \& Roshko (1974) in their figure 10, corresponding to

$$
\lambda \equiv\left(U_{1}-U_{2}\right) /\left(U_{1}+U_{2}\right) \simeq 0 \cdot 65,
$$

and very close to the result at a comparable velocity ratio obtained by Spencer \& Jones (1971).

\section{Flow-visualization results}

As mentioned previously, a particular advantage of conducting such an experiment in a water tunnel is the simplicity of flow visualization using dye injection. Also, the relatively low velocities required and the large dimensions of the facility permit simple photographic techniques.

It would probably have been advantageous to flood the whole low-speed side with dye so as to label one stream completely. The amount of dye required, however, would have contaminated the closed tunnel circuit very quickly. Consequently, it was decided to inject dye locally from the base of two twodimensional airfoil sections spanning the tunnel test section at zero angle of attack. Commercially available airfoil-shaped tubing $(12 \mathrm{~mm}$ chord, $3 \mathrm{~mm}$ thickness) was used with thin slots milled in the base over the central third of the span. These dye injectors were placed symmetrically just outside the shearlayer edges, about $30 \mathrm{~cm}$ downstream of the splitter plate's trailing edge. By changing their position it was possible to ascertain that they had no effect on the large structure being observed.

The flow visualization was carried out with a free-stream velocity on the high-speed side of $100 \mathrm{~cm} / \mathrm{s}$, without the lucite top (to permit the injectors to be installed). This velocity was chosen because it was the highest for which the corresponding velocity on the low-speed side was subcritical $(<23 \mathrm{~cm} / \mathrm{s})$ with respect to surface waves, resulting in a quiescent free surface. Photographs obtained by injecting from the high-speed side and from the low-speed side respectively are shown in figures $4(a)$ and $4(b)$ (plate 1 , where flow is from right 
to left and the high-speed layer is at the bottom; see also figure 2). The large structures are quite evident. The Reynolds number based on the velocity of the high-speed side and the distance from the centre of the photographs to the trailing edge of the splitter plate was equal to $2 \times 10^{6}$.

With the aid of the flow visualization it was possible to observe the interactions between the large structures. Winant \& Browand (1974) described a process of pairing (or tripling) of large structures at much lower Reynolds numbers. Two (or three) structures would come close together and start rotating around each other, while retaining their respective identities, eventually coalescing to form a new, larger structure. While this process of pairing, as described by these authors, was also observed at higher Reynolds number in our shear layer, a more violent process seemed also to be responsible for the reduction of the number of identifiable structures as they progressed down the shear layer. This process may be seen more clearly in the motion pictures and by direct observation and is perhaps better described as 'tearing'. A large structure will occasionally find itself in the vicinity of another, or in between two others, in whose straining field it disintegrates. The fluid associated with the structure that disintegrates becomes part of the collective motion of the neighbouring structure (or structures). This latter stage of the amalgamation process is associated with a considerable increase in turbulent activity (in a Lagrangian frame). This picture of 'tearing' is very similar in many respects to the one proposed by Moore \& Saffman (1975).

The flow visualization also allowed us to observe the entrainment mechanism. In a frame of reference moving with the convection velocity of the large structure, fluid appeared to be entrained into the shear layer from both the low- and the high-speed side in a symmetric fashion. One can also conclude that the initial stages of this mechanism resemble a process of engulfment in which the velocity induced in the two streams by the large structure (approximately a two-dimensional vortex) causes the freshly entrained irrotational fluid to penetrate well into the layer. Dye labelling fluid from one side can be seen to cross all the way into the other side before any small-scale mixing occurs. This is perhaps more clearly evident in figure 5 (plate 2), in which dyes of different colours have been injected from each side at the same time. Note the similarity between this picture and the photographs of Brown \& Roshko (1974).

Once inside the layer, the freshly entrained fluid appears to be associated with the irrotational part of the large-scale motion of the nearest large structure and remains distinguishable during that large structure's lifetime. However, it seems to mix very rapidly and down to very small scales throughout the layer once the identity of the particular large structure is lost by some means or other. The latter behaviour may be more clearly seen in the movies and by direct observation.

To investigate this final stage of the mixing process more directly and to obtain a qualitative feeling for the rapidity of the subsequent molecular mixing, a different scheme was employed. The blue dye was replaced with dilute nitric acid containing bromothymol blue, a pH indicator, and the red dye was replaced by (colourless) ammonium hydroxide solution. The bromothymol blue changes 
from deep orange in an acidic environment to deep blue in the alkali, the change occurring close to a $\mathrm{pH}$ of 7. A photograph of this change occurring in the mixing layer, as the entrained acid reacted with the entrained alkaline solution, is shown in figure 6 (plate 2). Note, from figures $4(a), 4(b)$ and 5, that the centre of the shear layer is located approximately halfway between the two bottom dots on the right-hand side of the picture (threaded inserts in the tunnel's lucite side walls). The acid in the picture (orange) has already entered the shear layer $\left(\delta_{\mathrm{viz}}\right.$ of Brown \& Roshko 1974) and can be seen to participate in the large structure's motion. It does not react (become blue), however, until later. It can be seen that, at these Reynolds numbers, the reaction, once it begins, occurs almost instantaneously, indicating the high efficiency of small-scale mixing in the latter stages of entrainment, and consistent with the observations with the inert dyes described above. If the decrease in the characteristic scales of the entrained fluid occurred by means of a gradual process, regions of unreacted fluid would have been discernible for a greater distance. The length scale of this gradual process would have been of the order of the large-structure spacing. This is not the case as can be seen in figure 6 (plate 2).

Thus it would appear that the processes of entrainment and fine-scale mixing, at high Reynolds numbers, are almost distinct and separate stages. This observation is also in agreement with some new detailed measurements obtained in the Brown \& Roshko (1974) gas mixing apparatus by Brown \& Konrad (to be published). It is also interesting to note that the details of the initial stages of the entrainment process, described above, are almost identical to the observations of Winant \& Browand (1974), at much lower Reynolds numbers.

In this general context, the dominant role of the dynamics and interaction of the large structure in the overall mechanism that eventually brings the two fluids into intimate contact becomes apparent. It is clear that any theoretical attempts to model the complex mixing process in the shear layer must take this ubiquitous large structure into account.

\section{Velocity measurement instrumentation and data processing}

The streamwise velocities were measured using a single-particle laser-Doppler velocimeter (Dimotakis \& Lang 1974) in the reference-scatter mode. See figure 7. The apertures at the receiving end were chosen to include the full beam width of the reference beam. Thus the scattering angle $\theta$ was defined as the angle subtended by the scattering beam axis and the reference-beam axis, the reference beam serving as the spatial filter (Siegman 1966; Mayo 1970). It was measured to be $\theta=4 \cdot 694^{\circ}$, resulting in a fringe spacing in the overlap volume of (heliumneon laser, $\lambda=0.6328 \mu \mathrm{m}$ )

and a Doppler constant of

$$
\lambda / 2 \sin \frac{1}{2} \theta=7 \cdot 725 \mu \mathrm{m}
$$

$$
\frac{2}{\lambda} \sin \frac{\theta}{2}=1.295 \frac{\mathrm{kHz}}{\mathrm{cm} / \mathrm{s}} \text {. }
$$

The actual velocity measurement consisted of measuring the time of flight of a single scattering particle for a distance corresponding to eleven fringe planes 


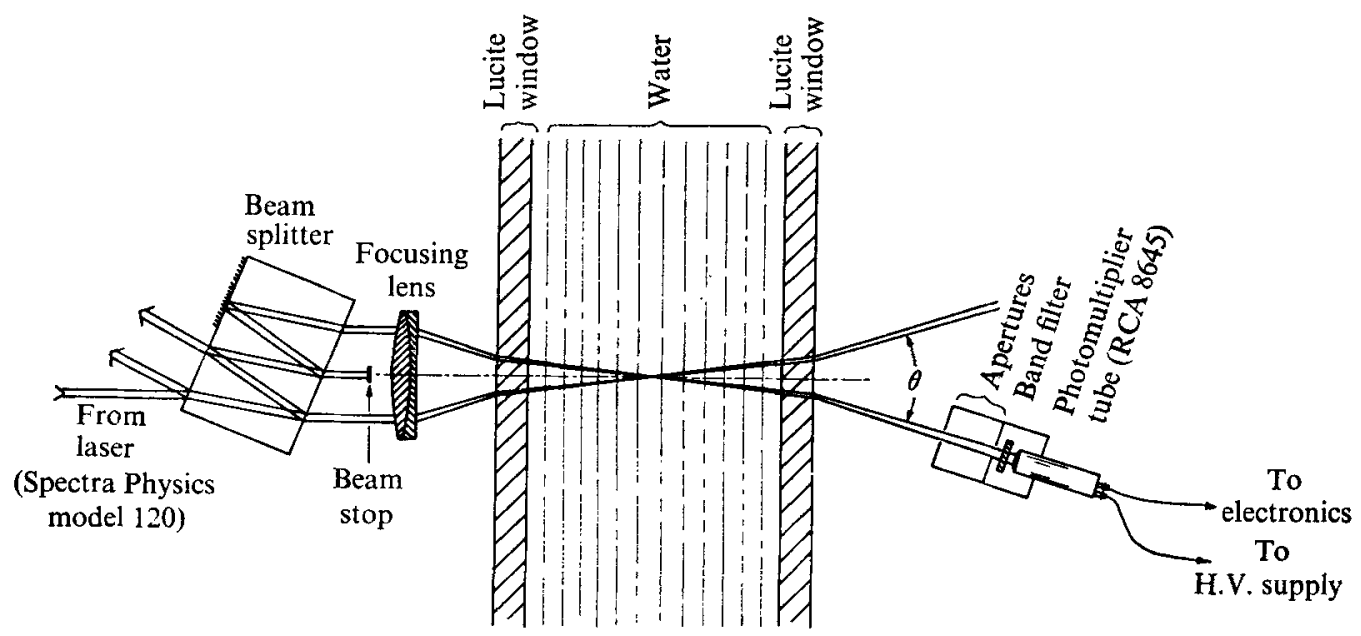

FiguRe 7. Laser-Doppler reference-scatter optics.

(ten intervals). The real time of each event was recorded by reading a freerunning counter, counting a crystal-controlled time base. The average data rate was monitored and could be controlled by adjusting the level that the amplitude of the signal burst due to a single particle had to cross to be processed. As the level was raised, fewer particles could meet this criterion and the data rate decreased (Dimotakis 1976). The limitation on the velocity measurement accuracy appeared to be connected with non-uniformity of the fringe-plane spacings in the focal volume. This was due to optical aberrations introduced by the $10 \mathrm{~cm}$ thick lucite water-tunnel windows, through which the measurement had to be made. In the absence of such aberrations, it can be shown that no ambiguity exists between the measured time of flight of a single scattering particle, for a distance corresponding to a fixed number of fringe planes, and the velocity component of the particle perpendicular to the fringe planes.

The time of flight and real time for each particle are coded into a single 32 bit word and stored in a $2 \times(1024 \times 32)$ high-speed buffer memory and subsequently recorded on a Kennedy $1600 / 360$ incremental tape recorder, operated in the slewing mode. A block diagram of the signal processing and data acquisition appears in figure 8 .

The resulting tape is processed, record by record, to yield the data

$$
\left\{\left(t_{i}, u_{i}\right), \quad i=1, N\right\},
$$

where $t_{i}$ is the real time of the crossing of the $i$ th particle, $u_{i}$ its streamwise velocity component and $N=1024$. Sample data are presented in figures $9(a)$, $9(b)$, and 10. Each figure represents one data record consisting of 1024 individual measurements as described above.

In computing the various stochastic quantities of interest from such data, care should be exercised in the choice of algorithms, in view of the inherent sampling bias of this method of measurement (McLaughlin \& Tiederman 1973; 


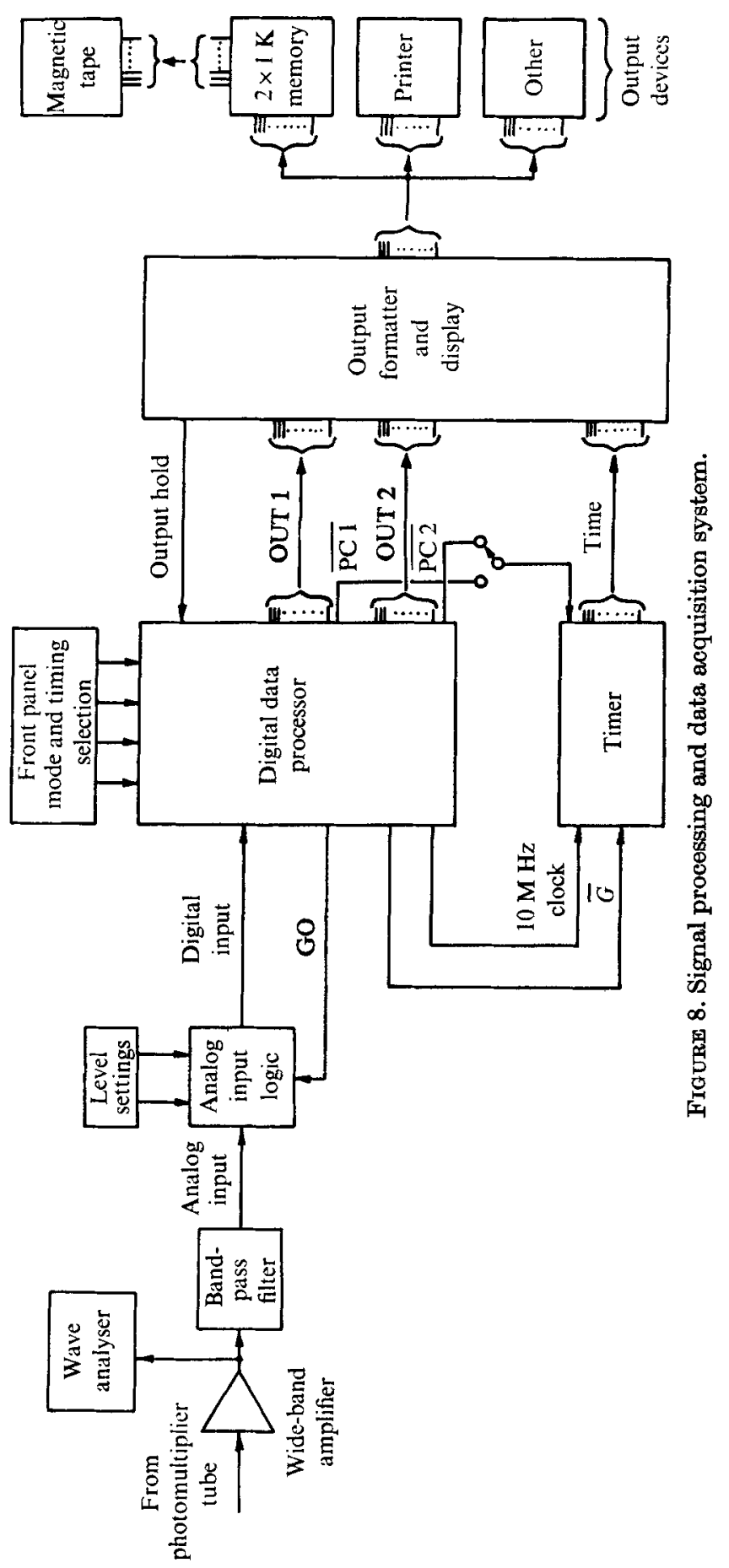



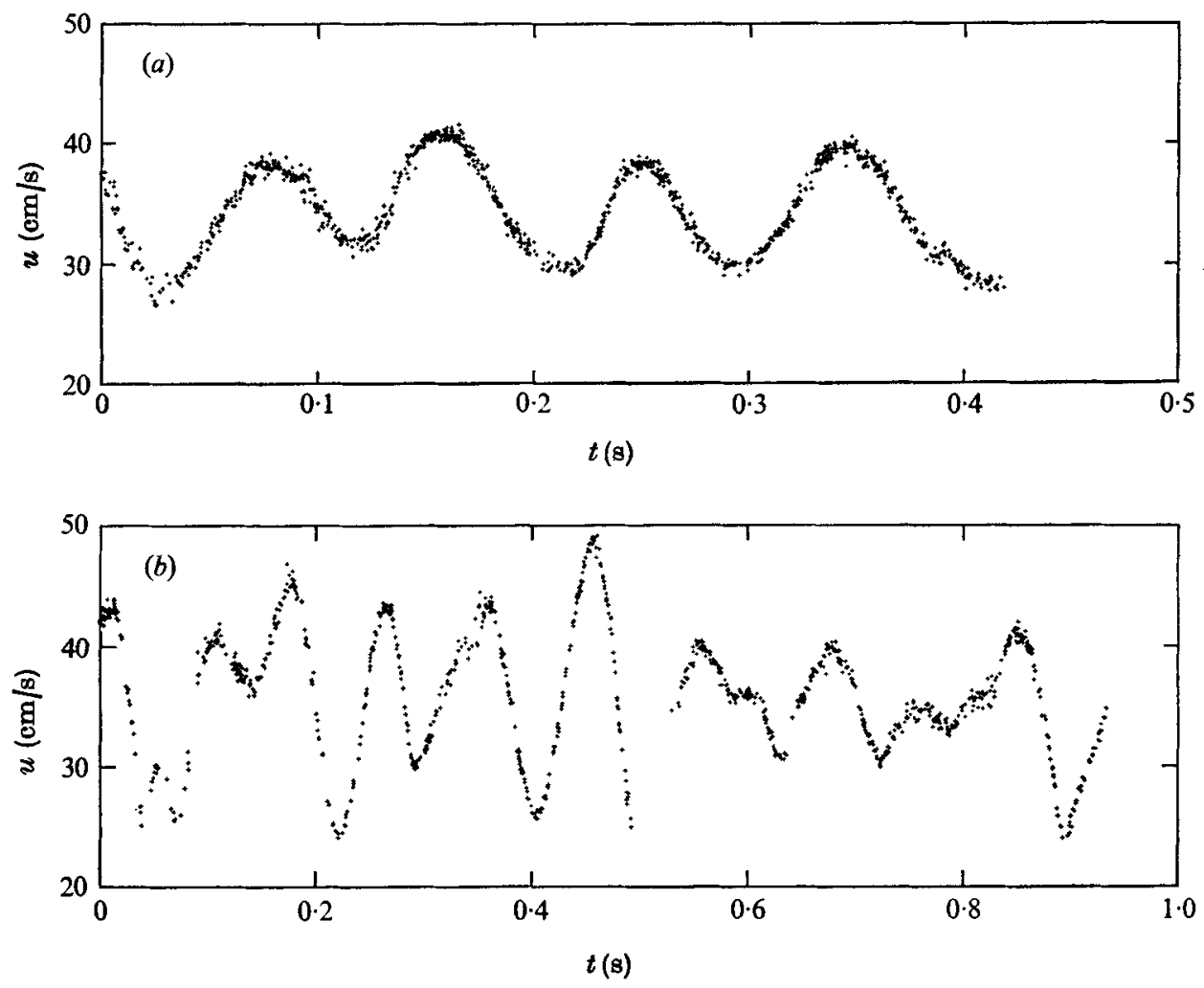

FTGURE 9. Velocity records taken at $U_{1}=165 \mathrm{~cm} / \mathrm{s}$, low-speed edge. (a) $T=0.419 \mathrm{~s}$, (b) $T=0.934 \mathrm{~s} . U_{\mathrm{z}}=35 \mathrm{~cm} / \mathrm{s}, x=30.48 \mathrm{~cm}, y=-3.53 \mathrm{~cm}, \eta-\eta^{*} \equiv\left(y-y^{*}\right) /\left(x-x_{0}\right)=-\frac{1}{8}$.

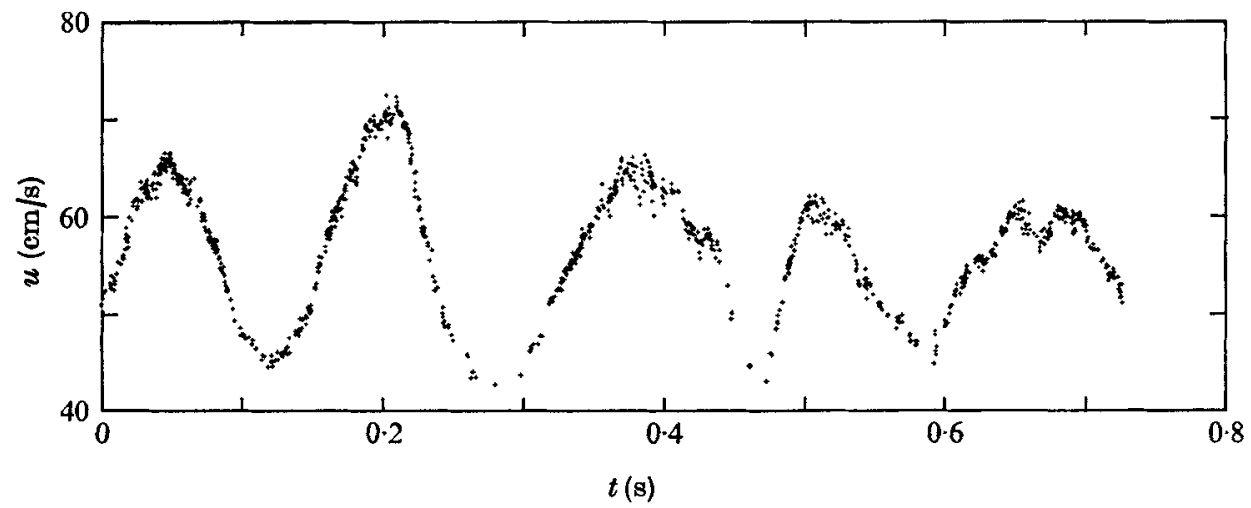

FIGURE 10. Velocity record at low-speed edge of shear layer for $U_{1}=330 \mathrm{~cm} / \mathrm{s}$, $T=0.726 \mathrm{~s} . U_{2}=59 \mathrm{~cm} / \mathrm{s}, x=91 \cdot 4 \mathrm{~cm}, y=-12 \cdot 1 \mathrm{~cm}, \eta-\eta^{*}=-\frac{1}{8}$. 
Barnet \& Bentley 1974; Dimotakis 1976). In particular, algorithms approximating time integrals, as opposed to (unweighted) ensemble averages, should be preferred. For example, mean velocities are best computed as

$$
\bar{u}=\frac{1}{T} \sum_{i=2}^{N} u_{i} \delta t_{i}
$$

where

$$
T=t_{N}-t_{1}, \quad \delta t_{i}=t_{i}-t_{i-1} \text {. }
$$

This estimate can differ by a few per cent, depending on the details of the sampling process and the velocity distribution function, from the biased ensemble average

(Dimotakis 1976).

$$
\langle u\rangle_{b}=\frac{1}{N} \sum_{i=1}^{N} u_{i}
$$

The errors can be more serious in computing the autocorrelation function, where errors resulting from subtracting the incorrect mean can be misinterpreted as correlations for large time shifts. The autocorrelations presented in the subsequent sections were computed in the following manner. Any small parabolic trend was removed from each record, i.e.

$$
u_{i}^{\prime}=u_{i}-\bar{u}-\left(b_{0}+b_{1} t_{i}+b_{2} t_{i}^{2}\right),
$$

where $\bar{u}$ was computed in terms of (1) and $b_{0}, b_{1}$ and $b_{2}$ were chosen to satisfy the condition

$$
\sum_{i=2}^{N}\left[u_{i}-\bar{u}-\left(b_{0}+b_{1} t_{i}+b_{2} t_{i}^{2}\right)\right]^{2} \delta t_{i}=\min
$$

The autocorrelation function was then computed by the algorithm

$$
R_{u u}(\tau)=\frac{1}{t_{M}-t_{1}} \sum_{i=2}^{M} u_{i}^{\prime} u^{\prime}\left(t_{i}+\tau\right) \delta t_{i}
$$

where $M$ is the largest index such that $t_{M}+\tau<t_{N}$ and $u^{\prime}\left(t_{i}+\tau\right)$ is the linearly interpolated value of the velocity at $t=t_{i}+\tau$, i.e.

where

$$
u^{\prime}\left(t_{i}+\tau\right)=u_{j-1}^{\prime}+\left(t_{i}+\tau-t_{j-1}\right) \delta u_{j}^{\prime} / \delta t_{j}
$$

$$
t_{j-1}<t_{i}+\tau<t_{j}, \quad \delta t_{j}=t_{j}-t_{j-1}, \quad \delta u_{j}^{\prime}=u_{j}^{\prime}-u_{u-1}^{\prime} .
$$

The autocorrelation function was never computed for shifts $\tau$ corresponding to less than the mean sampling interval

$$
\tau_{s}=T /(N-1)
$$

In addition, only records containing more than four large structures but less than thirty were used. The upper limit of thirty was imposed because of the finite number of points per record (1024). Within these two limits there were enough structures in the record for the fundamental frequency to be determinable and enough measurements per large-structure cycle to justify the numerical timeintegration scheme (Dimotakis 1976).

A free-stream velocity of $U_{1}=330 \mathrm{~cm} / \mathrm{s}$ was the highest velocity at which quantitative measurements were recorded. Efforts to record data at higher 
velocities were frustrated by an unexpected difficulty. Small particles, rust, mud and general debris were picked up from other parts of the circuit by the higherspeed flow, seriously impairing visibility in the test section and rendering the laser-Doppler velocity measurements difficult. An improved filtration system that has since been installed will permit measurements at the higher velocities that can be attained in this facility (an increase greater than a factor of two in the Reynolds number).

\section{Velocity measurements and autocorrelation functions}

Most of the velocity measurements were made just outside the shear layer, corresponding to a similarity co-ordinate of

$$
\eta-\eta^{*}=\left(y-y^{*}\right) /\left(x-x_{0}\right) \sim \pm \frac{1}{8} .
$$

$x_{0}$ and $y_{0}$ are the co-ordinates of the virtual origin of the shear layer and

$$
\eta^{*}=\left(y^{*}-y_{0}\right) /\left(x-x_{0}\right)
$$

is the similarity co-ordinate of the ray on which the mean velocity is given by

$$
U\left(\eta^{*}\right)=\frac{1}{2}\left(U_{1}+U_{2}\right)
$$

As can be seen from the velocity profiles in figure 3 , these measurement locations correspond to a local mean velocity that is almost equal to that of the corresponding free stream $\left(U_{1}\right.$ or $\left.U_{2}\right)$. One might conclude from this observation that the amplitude of the local fluctuations in the streamwise velocity would be small. However, the sample data presented in figures $9(a), 9(b)$ and 10 show quite the contrary. Several observations and conclusions can be made from these data samples.

One would be forced to deduce, from such data alone, the existence of a large organized structure within the shear layer. These fluctuations, measured in the neighbouring free stream, could not have been caused by small, randomly distributed and randomly oriented domains of high turbulence activity within the shear layer. The effect of many such small domains would have been essentially cancelled by the superposition of the various uncorrelated disturbances and attenuated by the distance between the measurement location and the corresponding region in the shear layer, as scaled by the characteristic size of these domains. In fact, the effect appears far from random and, as we shall show shortly, is characterized by a well-defined average periodicity.

The second observation is related to the magnitude of these large-scale fluctuations. It can be seen that, for the sample measurements at the low-speed edge of the shear layer, the instantaneous value of the velocity is well below that of the undisturbed free stream for a very high fraction of the time. The corresponding behaviour is found on the high-speed side of the layer. It is perhaps difficult to account for this behaviour without taking into consideration the vortical nature of the large structure that is apparent in the flow-visualization photographs and motion pictures discussed in \$ 3. Evidently these fluctuations represent the potential flow induced by the large structures as they are convected 
downstream. These extend to the as yet unentrained fluid in the vicinity of the shear layer. With this picture in mind, one can also appreciate that the amplitude of the perpendicular velocity fluctuations (along the $y$ axis) would be of the same order of magnitude. This latter component is, of course, more directly connected with the entrainment process. The amplitude of these potential fluctuations agrees quite well with the amplitudes measured by Wygnanski \& Fiedler (1970) at the corresponding value of the reduced co-ordinate, on the high-speed side of their $U_{2}=0$ shear layer; they found good agreement with the 1 -power attenuation law suggested by Phillips (1955).

The third observation is related to the large variability between successive records. Within the shorter record of figure $9(a)$, four almost identical structures have evidently been convected past the measuring station. By contrast, within the longer record of figure $9(b)$ (identical conditions except for a higher minimum burst amplitude level in the LDV signal processor; see previous section) evidence of various stages of amalgamation between structures can be inferred. For example, the peaks around $0.10 \mathrm{~s}$ and $0.20 \mathrm{~s}$, and $0.75 \mathrm{~s}$ and $0.85 \mathrm{~s}$ suggest that a larger structure is being formed by the amalgamation of two neighbouring structures. The time interval of approximately $0.10 \mathrm{~s}$ between those two events can be seen to be the characteristic period of the fluctuations for the data in both figure $9(a)$ and figure $9(b)$. Closer inspection of the data record in figure $9(b)$ will also reveal evidence of other stages of the amalgamation process. The data record in figure 10 represents measurements taken $90 \mathrm{~cm}$ downstream of the splitter plate at a free-stream velocity on the high-speed side of $U_{1}=330 \mathrm{~cm} / \mathrm{s}$. A simple scaling calculation will reveal that the basic periodicity in this record is consistent with that of the previous two. The Reynolds number based on $U_{1}$ and $x$ for the data in figure 10 is equal to $3 \times 10^{6}$ and is the highest at which quantitative data were recorded.

To examine the general periodic character of these data in a more quantitative fashion, the autocorrelation function of the velocity, given by

$$
R_{u u}(\tau)=\overline{u^{\prime}(t) u^{\prime}(t+\tau)}
$$

was computed for each record (see $\S 4$ ).

Figures $11(a)-(d)$ show the autocorrelation function of the streamwise velocity fluctuations, ensemble averaged over several records. The measurements were taken at the low-speed edge of the shear layer at $x=15,30,60$ and $90 \mathrm{~cm}$ downstream of the splitter plate. The free-stream velocity on the high-speed side was $U_{1}=165 \mathrm{~cm} / \mathrm{s}$.

To render the similarity behaviour of the fundamental periodicity more explicit, the time lag $\tau$ is scaled by the convection time

$$
\tau_{c}=\left(x-x_{0}\right) / U_{c}
$$

and plotted as a dimensionless variable

$$
\tau / \tau_{c}=\tau U_{c} /\left(x-x_{0}\right)
$$

$U_{c}$ is the convection velocity for the large structure, closely approximated by

$$
U_{c} \simeq \frac{1}{2}\left(U_{1}+U_{2}\right)
$$



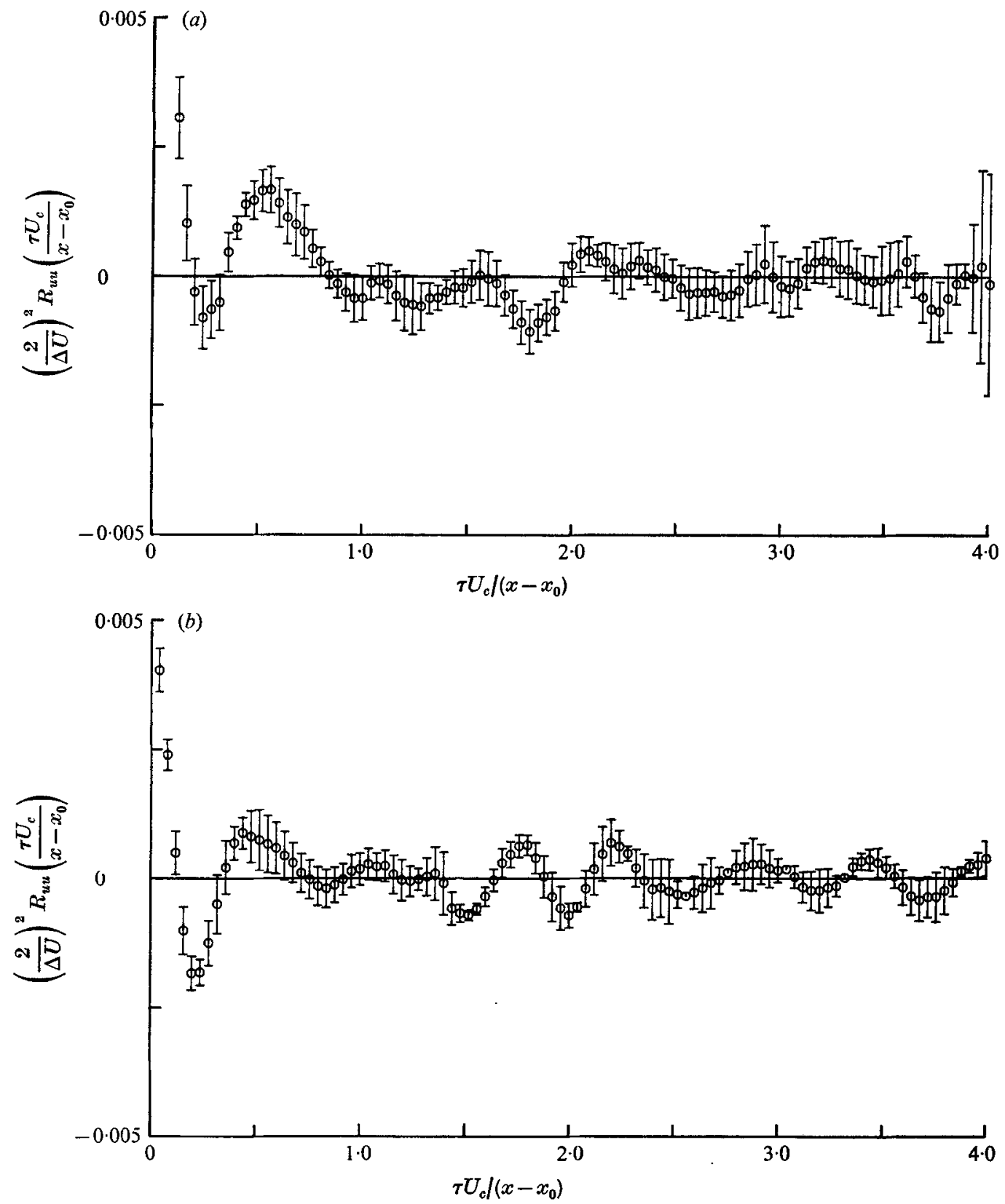

Frgures $11(a, b)$. For caption see page 550 .

for a shear layer of uniform density. The resulting values of the autocorrelation function are scaled by $\left(\frac{1}{2} \Delta U\right)^{2}$, where $\Delta U$ is the velocity difference across the shear layer $\left(\Delta U \equiv U_{1}-U_{2}\right)$. Note that $R_{u u}(0)=\overline{u^{\prime 2}}$. A perfectly coherent sinusoidal velocity fluctuation whose upper and lower amplitude limits are given by $U_{1}$ and $U_{2}$ possesses an autocorrelation function which, when scaled in this fashion, is a cosine oscillating between $\pm 1 \cdot 0$. The plotted error bars represent 

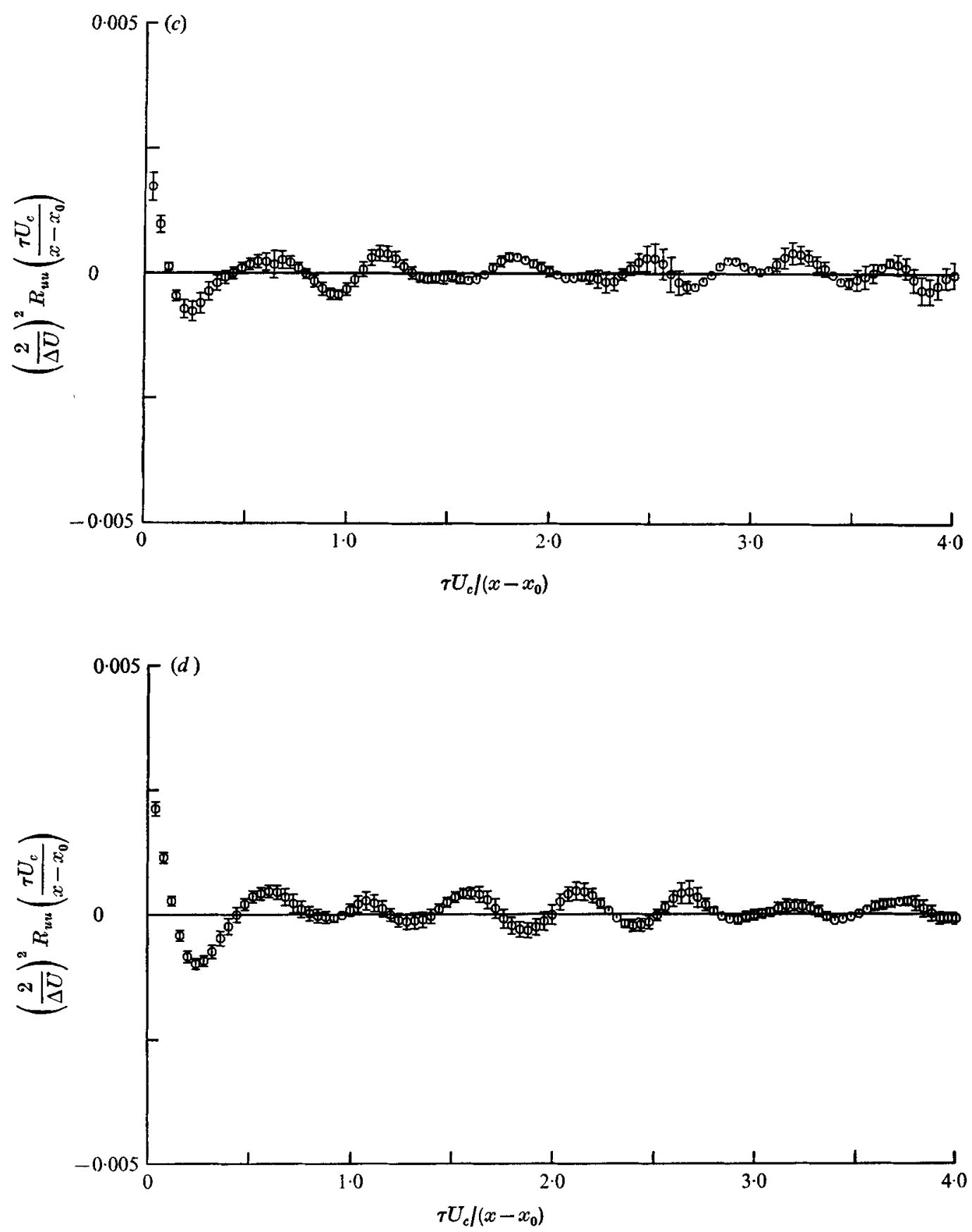

FIGURE 11. Autocorrelation function. $U_{1}=165 \mathrm{~cm} / \mathrm{s}, \eta-\eta^{*}=-\frac{1}{8}$. (a) $x=15 \mathrm{~cm},(b) x=30 \mathrm{~cm},(c) x=60 \mathrm{~cm},(d) x=90 \mathrm{~cm}$. 


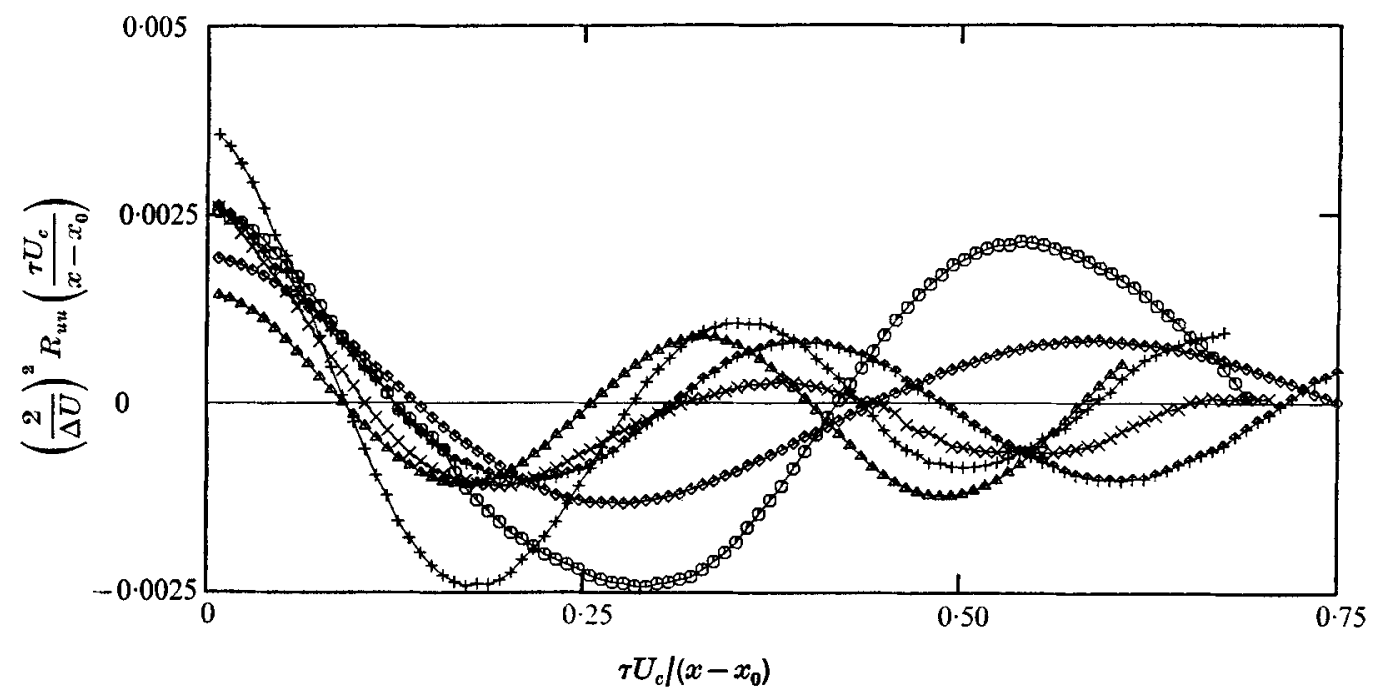

Figure 12. Six estimates of the autocorrelation function at $U_{1}=165 \mathrm{~cm} / \mathrm{s}, x=30 \mathrm{~cm}, U_{2}=35 \mathrm{~cm} / \mathrm{s}, \eta-\eta^{*}=-\frac{1}{8}$.

the standard deviation of the ensemble-averaged values of the autocorrelation function for each value of $\tau U_{c} /\left(x-x_{0}\right)$. The magnitude of these error bars is not the result of measurement uncertainties or computational errors but rather a measure of the variability between autocorrelation functions computed from consecutive data records. To illustrate this point, six estimates of the autocorrelation function computed from data records of measurements taken at $x=30 \mathrm{~cm}$ are plotted in figure 12 . The curve in figure 12 labelled by the crosses was computed from the data in figure $9(b)$. Each curve is smooth and well defined, yet both the period and the amplitude of the calculated autocorrelation functions can be seen to vary over a large range of values. A discussion of this important aspect of the data will be presented later on.

The most notable feature of the computed autocorrelation functions is that the fundamental periodicity of the velocity fluctuations appears to be consistent with the expected similarity scaling. If we define the fundamental period $\tau_{0}$ as twice the time interval before the first minimum of the autocorrelation function, we expect that, for a given $U_{1} / U_{2}$,

$$
\tau_{0} U_{c} /\left(x-x_{0}\right)=\text { constant }
$$

for all $x, U_{1}$ and $U_{2}$. All the estimates of this quantity, resulting from measurements at $U_{1}=31 \cdot 4,165$ and $330 \mathrm{~cm} / \mathrm{s}$ and $x=15,30,60$ and $90 \mathrm{~cm}$ at both the low-speed and the high-speed edge of the shear layer, are consistent with the inequality $\left(U_{1} / U_{2} \simeq 5\right)$

$$
0.40<\tau_{0} U_{c} /\left(x-x_{0}\right)<0.50 .
$$

The spacing between the structures, given by

$$
l=\tau_{0} U_{c},
$$


is more appropriately scaled by the local vorticity thickness $\delta_{\omega}$, defined in terms of the maximum slope thickness of the mean velocity distribution, i.e.

$$
\frac{1}{\delta_{\omega}} \equiv\left(\frac{1}{\Delta U} \frac{\partial U}{\partial y}\right)_{\max }
$$

Using the experimentally determined range of values for $\delta_{\omega}$ [see (1)], we have

$$
3 \cdot 1<\tau_{0} U_{c} / \delta_{\omega}<5 \cdot 0,
$$

in reasonable agreement with the values inferred by Brown \& Roshko (1974) from the data of Spencer \& Jones $\left(1971 ; \tau_{0} U_{c} / \delta_{\omega} \sim 3 \cdot 8\right)$ and Winant \& Browand $\left(1974 ; \tau_{0} U_{c} / \delta_{\omega} \sim 3 \cdot 3\right)$, and also consistent with the stability criterion for a single vortical structure of Moore \& Saffman (1975). Basing their argument on the stability of a vortex in the presence of the distorting field of its neighbours, the last authors derive a stability criterion for a single structure that requires

$$
l \gtrsim \mathbf{3 \cdot 5} \delta_{\omega} \text {, }
$$

where $l$ is the spacing between vortices and $\delta_{\omega}$ is the local maximum slope thickness.

The agreement between the fundamental periods corresponding to measurements at the low-speed edge and the high-speed edge is worth noting. The local velocities there differ by almost a factor of five. It would be very difficult to explain an Eulerian frequency that is the same on both sides without recognizing the existence of a structure whose dimensions are of the order of the full shearlayer width and which moves as a unit with a convection velocity of its own.

The second result that emerges from these data is related to the surprisingly long correlation times associated with the large-structure dynamics. The autocorrelation function retains its oscillatory character for time lags that are longer than any obviously relevant time scale. The large structures amalgamate and disappear as identifiable entities within a distance of the order of their mutual separation (Brown \& Roshko 1974), and maintain a spacing proportional to $x-x_{0}$, on the average. One would not expect any coherence beyond the identifiable lifetime of each structure. Consequently, the oscillations in the autocorrelation function should have decayed after, at most, two structure periods, corresponding to

$$
\tau U_{c} /\left(x-x_{0}\right) \lesssim 1
$$

[see (10)]. Since $\left(x-x_{0}\right) / U_{c}$ is also the convection time required for a disturbance associated with the large structure to traverse the distance $x$, the upper bound given by (13) is the largest obvious time scale for the problem. Yet, within the experimental confidence limits, the data suggest the existence of memory times much longer than this upper bound. It is as if the large-structure phase is not completely destroyed in the amalgamation process, but remains coupled to the phase of the structures formed subsequently.

The third notable feature of the computed autocorrelation functions is related to the decrease in the standard deviation of the ensemble averages as $x$ increases. The autocorrelation functions computed from each record of measurements at 
the furthest downstream location agree reasonably well with each other. This is in marked contrast to the estimates for the measuring stations closer to the splitter plate, an example of which is shown in figure 12. In this respect, similarity scaling appears totally inadequate in explaining the dependence on $x$ of the variability in the estimates of the autocorrelation functions.

It is perhaps surprising that the well-defined oscillations in the estimates of the autocorrelation function have not been found previously. Several reasons may be responsible for this discrepancy. The most important of these, we believe, is the choice of the location of the measuring station. The velocity fluctuations were measured just outside the turbulent region of the shear layer $\left(\left|\eta-\eta^{*}\right| \sim \frac{1}{8}\right)$. At this location, the rapid, high intensity, small-scale fluctuations in the shear layer do not have any significant effect, the intervening fluid acting like a low-pass filter. The situation is akin to the case of the wake of a cylinder. The best place to observe the vortex shedding frequency is just outside the wake. Estimates of the autocorrelation function for small time lags computed from measurements at the centre of the shear layer $\left(\eta=\eta^{*}\right)$ have amplitudes for short time lags about ten times larger than the ones in figure 11. Even though the oscillatory nature for longer time lags can still be seen, it is appreciably less well defined. We believe that this is a computational difficulty associated with a broader spectrum and a much higher mean-square fluctuation level. The quasiperiodic nature of the signal was still quite apparent when the velocity measurements were plotted directly versus time. A second important reason becomes apparent if one reflects on the (vector) induced velocity of the vortical structures. If the structures were regular vortices with their cores perfectly lined up along an axis, the streamwise velocity perturbations on that axis would vanish. While these are important questions to be resolved, a more detailed study was not undertaken because of data-acquisition limitations at the time. Recent improvements, however, will allow measurements in the turbulent region for the purposes of clarifying these issues and investigating the small scales.

\section{The effect of the initial conditions}

There is a growing body of evidence that suggests that the initial state of the shear layer may also be important in determining the growth rate of the linear region. There is reason to believe for example that the $30 \%$ difference between the growth rates measured by Liepmann \& Laufer (1947) and by Wygnanski \& Fiedler (1970) may be attributable to a tripping device placed in the boundary layer by the latter, upstream of the (real) origin of the shear layer. Batt (1975) could reproduce the Liepmann \& Laufer (1947) data or the Wygnanski \& Fiedler (1970) data by tripping or not tripping the boundary layer in the same apparatus.

A possible explanation of the importance of the initial conditions may lie in an analysis by Winant (1972) of the shear-layer growth, on the basis of the vortex pairing mechanism. He concludes that the details of the initial vorticity distribution within the structures and the irregularities in the initial lateral displacement of the vortices have a definite effect on the subsequent growth rate. 
The situation is well summarized by the collection of data in figure 10 of Brown \& Roshko (1974), where growth rates measured by several experimenters are plotted as a function of the dimensionless ratio $\lambda=\left(U_{1}-U_{2}\right) /\left(U_{1}+U_{2}\right)$. If similarity reasoning is applicable, the shot gun appearance of the plotted points, within a relatively wide band of values, is something of an embarrassment. Nevertheless it is not possible to say, at present, whether this departure from similarity is the result of the persistence of the effects of the initial conditions or experimental difficulties in generating a truly self-preserving shear layer. The latter difficulty could be a consequence of the very large dimensions required. The two possibilities are, of course, not necessarily mutually exclusive.

Bradshaw (1966), in his investigation of the transition region of the shear layer, concluded that a streamwise extent of as many as 1000 initial momentum thicknesses may be required before similarity can be attained. A similar estimate can be obtained if one requires that the large-structure spacing be at least ten times larger than the spacing of the initially amplified disturbance (Roshko 1974). This condition could, in fact, explain the large difference in the variability of the autocorrelation functions computed for a $U_{1}=165 \mathrm{~cm} / \mathrm{s}$ at $15 \mathrm{~cm}$ and $30 \mathrm{~cm}$ downstream of the splitter plate (figures $11 a, b$ ) and those measured further downstream. In this case, one thousand momentum thicknesses corresponds to $x=40 \mathrm{~cm}$. It is interesting to note that the mean large-structure spacing seems to have attained its self-preserving value as early as $15 \mathrm{~cm}$ downstream of the splitter plate.

It may be, however, that the estimate of a thousand momentum thicknesses for full similarity to be attained is actually low. This becomes plausible if we consider the large-structure amalgamation scaling. If we assume that the spacing between large structures doubles after each interaction, then the number of interactions (tearings, pairings, etc.) between the shear-layer origin and $x$ is given by

$$
m(x)=\log _{2}\left(x / l_{0}\right)
$$

where $l_{0}$ is the spacing of the initial disturbance. One thousand momentum thicknesses corresponds to a value of $m$ between 3 and 4, hardly a large number. Efforts to allow the shear layer to 'forget' the initial conditions over a large downstream distance should be evaluated in terms of the sobering scaling of (14). The expected total number of interactions in this experiment (for $U_{1}=165 \mathrm{~cm} / \mathrm{s}$ ) within the effective test section $0<x<L \sim 2 \mathrm{~m}$ is approximately given by $m(L) \sim 6$.

A second factor which would be expected to play a role is related to the distribution of vorticity within the large structure. There is reason to believe that it affects the dynamies of the large-structure growth and amalgamation (Winant 1972; Moore \& Saffman 1975). In terms of the radius of gyration $r_{G}$ of the vorticity distribution, given by

$$
r_{G}^{2} \equiv \frac{1}{\kappa} \iint r^{2} \omega(r, \phi) r d r d \phi
$$

where $\kappa$ is the total circulation

$$
\kappa \equiv \iint \omega(r, \phi) r d r d \phi
$$


a shape parameter can be defined as $\beta \equiv 2 r_{G} / \delta$, where $\delta$ is the diameter of the structure (cf. $\delta_{\mathrm{viz}}$ of Brown \& Roshko 1974), and is a measure of the concentration of vorticity within the structure ( $\beta=0$ corresponds to a point vortex). A 'young' structure in the layer, the result of one or two amalgamations (small $m$ ) will have a vorticity distribution closely related to the initial conditions and possibly different from that of an 'old' structure which has been formed as the result of several amalgamations (larger $m$ ). Consequently, in a finite apparatus, the 'linear' growth rate, for a shear layer of uniform density, would be a function of both $U_{1} / U_{2}$ and the vorticity distribution parameter $\beta$, i.e.

$$
d \delta_{\omega}(x) / d x \sim f\left(U_{1} / U_{2} ; \beta\right) .
$$

For a given set of initial conditions, the value of $\beta$ corresponding to a structure at $x$ would primarily depend on $m$, the structure's 'age'. The notion of the 'age' of a vortical structure was introduced by Owen (1970), who found a correlation between the decay of aircraft trailing vortices and a logarithmic measure of the vortex lifetime.

Thus the growth rate, which depends on the slowly changing shape parameter, would, in turn, also change slowly. The logarithmic dependence of $m$ on $x$, however, could easily have caused such an effect to have escaped detection within the extent of any single apparatus. This is quite plausible since, in turn, the dependence of the growth rate on the vorticity distribution shape parameter $\beta$ is probably also a weak one. For a fixed measuring station, however, the effect of changing $\beta$ by adding a 'tripping' wire could easily be noticeable (Batt 1975).

For a boundary layer that is initially laminar, the value of the initial largestructure spacing $l_{0}$ is of the order of ten boundary-layer thicknesses. A tripping wire, depending on the Reynolds number based on its own dimensions, may introduce length scales many times smaller. As a result, the vorticity distribution within the structure at the downstream measuring station could change because both the initial vorticity distribution would be altered and the 'age' $m$ would increase owing to the decrease in $l_{0}$.

These considerations suggest that the assumption that the initial conditions do not play a role in the downstream development of the 'linear' growth region may not be valid. The initial conditions may have an effect persisting for many logarithmic increments downstream by determining a shape factor on which, in turn, the growth rate depends weakly. If this is correct, the shear layer may be characterized by a 'linear' growth rate not because the initial conditions are forgotten so quickly but because they are forgotten so slowly. The 'linear' growth region should then be viewed as being defined not only in terms of a velocity ratio $U_{1} / U_{2}$ but also in terms of a weak dependence on such slowly changing characteristics as the distribution of vorticity within the large structure, which is largely determined by the initial conditions. 


\section{The long time scales}

The conjectures in the preceding section, if proven correct, could explain much of the apparent departure from similarity of the data available to date on the shear layer. They could not, however, explain the long memory time scales that were found in the autocorrelation functions, discussed in $\$ 5$.

The large variability in the estimates of the autocorrelation function from the records corresponding to the measurements closer to the splitter plate could be explained by the presence of characteristic times that are longer than those consistent with local scaling [i.e. $\left(x-x_{0}\right) / U_{c}$ ]. The fact that this variability does not appear in the measurements furthest from the splitter plate raises the possibility that time scales related to the total length of the apparatus are important.

For the structures at the furthest measuring station $(90 \mathrm{~cm}$ downstream of the splitter plate's trailing edge) only one more amalgamation is expected before the end of the test section ( $\sim 200 \mathrm{~cm}$ before wall effects are important). This means that characteristic times that are at most twice the local period can be found in the corresponding velocity fluctuations. Measurements recorded at this location, spanning a total of many local structure periods, have exhausted in some sense the possibilities for the velocity fluctuations. Consequently, estimates of the autocorrelation function computed from these records would be expected to be closer to the infinite time average for the process. In more conventional terms, in the problem of determining the spectrum for the process, each data record at larger $x$ is much longer than the reciprocal of the lowest frequency present. If a mechanism exists that can couple the longer periods of the downstream structures with the dynamics of the velocity fluctuations closer to the splitter plate, this would not be the case for the measurements at smaller $x$ 's. Recall that the records span time intervals proportional to $x$. At each measuring station, the record lengths were adjusted to include a given number of (local) large-structure cycles.

The existence of such a coupling scheme can be argued as follows. By considering the induced mean velocities outside the shear layer, we deduce that the ratio of the circulation around each vortical structure to the structure spacing must be given by

$$
\kappa / l=\Delta U
$$

where $\Delta U$ is the velocity difference across the layer $\left(\Delta U \equiv U_{1}-U_{2}\right)$. The largestructure spacing, however, increases in proportion to $x$ (Brown \& Roshko 1974; Winant \& Browand 1974), i.e.

$$
l /\left(x-x_{0}\right)=\alpha \sim \text { constant }
$$

where, for this experiment, $0.4<\alpha<0.5$ [inequality (10)]. Consequently, the expected circulation around a vortical structure will also increase with $x$, i.e.

$$
\kappa\left(x-x_{0}\right) \sim\left(x-x_{0}\right) \propto \Delta U .
$$

Therefore, even though the influence of a distant vortical structure on a given point decreases inversely with distance (infinite channel), the strength of distant 
vortices increases linearly with the downstream co-ordinate, and the effect does not vanish as the distance to the vortical structure increases.

The surprising conclusion is that all the structures downstream of a given point in a subsonic shear layer can influence the dynamics at that point. This could provide the coupling mechanism required to explain the observed variability in the estimates of the autocorrelation function. This coupling could in turn increase the importance of the initial conditions by essentially providing a feedback mechanism whereby an initial instability may be driven by or become synchronized with the downstream fluctuations. As the structure at the end of the test section leaves, it excites the next initial roll-up and keeps it in phase with the existing structures in the test section. In this manner the phase coherence can be preserved over times longer than $\left(x-x_{0}\right) / U_{c}$. The longest time scale available (i.e. $L / U_{c}$ ) determines the lowest frequency that will be found in the spectrum computed from data taken anywhere in the channel ( $L=$ channel length).

A closely related effect has been reported by Roshko (1967), who measured the velocity fluctuations in the transition region of a shear layer generated by a rearward-facing step. At a boundary-layer Reynolds number of $R e_{x} \sim 2 \times 10^{3}$, he found that the periodic fluctuations in the initial stages of the transition region were strongly modulated by a much lower frequency, the corresponding period being approximately ten times that of the local fluctuations. He speculates that the coupling synchronizes the oscillations in the location of the reattachment point, which are sustained by this feedback mechanism.

A more recent example of this feedback mechanism in the dynamics of the shear layer can be found in an experimental investigation of flow over a cavity (Sarohia 1976). In this case the free shear layer that originates at the upstream edge of the cavity reaches a self-similar mean velocity profile within fifteen initial momentum thicknesses. The feedback from the downstream edge of the cavity, however, is so strong as to impose well-defined resonant modes on the flow pattern. The shear layer over the cavity was found to grow linearly with $x$ after fifteen initial momentum thicknesses but at a rate which was a function of the ratio $L / \theta_{0}$, where $L$ is the total length of the cavity and $\theta_{0}$ is the initial momentum thickness. The situation is, of course, not quite equivalent because, in most cases, the cavity was not long enough for any large-structure amalgamations to have occurred. Nevertheless it is an example of a free shear layer where the finite extent of the layer plays an important role.

In view of this mechanism that couples the dynamics at a point in the shear layer to the structures downstream, the problem of a steady-state, subsonic, infinite shear layer must remain academic. An infinite shear layer would render the behaviour of the fluid at some station $x$ non-stationary in a stochastic sense. The feedback of ever decreasing frequencies from the downstream structures would make the estimation of the power spectrum of the fluctuations at $x$ impossible in principle. In practice, of course, the effect of the downstream feedback is reduced by three-dimensional effects (walls at $z= \pm \frac{1}{2} L_{z}$ ) or image flow (wall at $y=\frac{1}{2} L_{y}$ and/or $y=-\frac{1}{2} L_{y}$ ), so that the finite dimensions of the apparatus enter (logarithmically) to govern the dynamics. It appears, therefore, that an 
analysis of the behaviour of a subsonic two-dimensional shear layer cannot be local but must consider the full effect of the flow within the finite extent of the apparatus.

\section{Conclusion}

Using flow-visualization techniques, it was possible to verify directly and record photographically and in motion pictures the fact that the large structures that were observed in the shear layer at lower Reyholds numbers persisted to Reynolds numbers of $x U_{1} / \nu \sim 3 \times 10^{6}$. It was also possible to study the mechanism that eventually brings fluid elements originating from either side into intimate contact within the shear layer. It was found that the freshly entrained fluid is initially part of the irrotational motion of the large structure and remains largely unmixed during the lifetime of the associated structure. Mixing is very rapid, however, once it begins and appears to be intimately connected with the amalgamation processes of the large structure. This was verified directly by labelling the fluid being entrained from the two sides with an acid and a base respectively, and observing the chemical reaction through the change in colour of a $\mathrm{pH}$ indicator, pre-mixed with the acid. It now appears that entrainment across the shear-layer boundaries and fine-scale mixing with the rest of the fluid within those boundaries are two almost distinct stages of the overall process.

Using laser-Doppler velocimetry techniques, discrete measurements of the streamwise velocity component were made at the edges of the shear layer. From the computed autocorrelation functions of the streamwise velocity fluctuations, it was possible to assign a fundamental periodicity to the large structure that was found to be in accordance with the expected similarity scaling. Other quantitative features of the velocity data, however, do not appear to be consistent with expected similarity behaviour and anticipated time scales. To explain these features and published shear-layer data from other sources, two hypotheses are formulated.

It is argued that, as a result of the logarithmic dependence of the number of large-structure interactions (amalgamations) on the effective length of the shear layer, the initial conditions play an important role within the extent of any realizable experimental apparatus. Consequently, the growth rate of the shear layer and other related features are not expressible as universal functions of the classical dimensionless parameters, $U_{1} / U_{2}, \rho_{1} / \rho_{2}, M_{1} \equiv U_{1} / a_{1}$, etc. Second, it is observed that, whereas the induced velocity at a point in the shear layer owing to a vortical structure downstream diminishes inversely as the distance to the structure, the strength (circulation) of a vortical structure increases in proportion to the distance between the structure and the shear-layer origin. As a result, in a subsonic shear layer, all the structures downstream of a given point affect the flow at that point. Consequently, it becomes necessary to analyse the two-dimensional shear layer globally, taking into consideration the effects of the initial conditions and the finite extent of the flow apparatus. 
Even though we should be considered responsible for the results and ideas presented here, we should like to think of this work as the direct outgrowth of the collective activity of the GALCIT Fluid Mechanics Group. We are particularly indebted to Professor Anatol Roshko for his advice, guidance and critical ear throughout the experimental work and data analysis. Many thanks are due to Dr J. E. Broadwell, Professor D. E. Coles and Professor P. G. Saffman for many helpful arguments and assistance with the text. The success of the novel signal processing and data acquisition system is due, to a large extent, to the expert design and construction of the electronics by Mr Daniel Lang. We should also like to thank Mr Tom Ward for his cooperation and assistance in the operation of the GALCIT Free Surface Water Tunnel. One of us (P.E.D.) would also like to thank Professor I. J. Wygnanski for a short discussion in which he brought to our attention the results on the mixing-layer growth by Batt (1975). The work was supported by the Office of Naval Research (Fluid Dynamics Program Contract no. N00014-67-A-0094-0037 and Project SQUID Contract no. 4965-42) and the Caltech President's Fund Grant no. PF-075.

Note added in proof. Recent experimental investigations (Oster 1976) corroborate the long-term coherence of the shear-layer large structures observed in this experiment. More direct evidence supporting the presence of a feedback mechanism, hypothesized in this paper, was found in the form of a frequency of the order of $L / U_{c}$ ( $L=$ length of shear layer) in the spectrum of the velocity fluctuations at all values of the streamwise co-ordinate.

The effect of the initial conditions is as yet unclear. Oster (1976) finds that, in a $U_{2} / U_{1}=0$ shear layer, tripping the boundary layer increases the shearlayer growth rate, in agreement with Batt (1975), whereas at a $U_{2} / U_{1}=0.4$

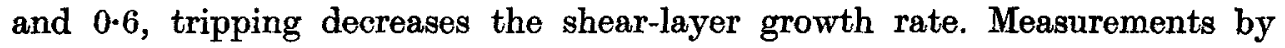
Browand \& Latigo (1976) on a $U_{2} / U_{1}=0 \cdot 23$ shear-layer, however, indicate that, even though tripping the boundary layer decreases the growth rate initially, the growth rate approaches that of the untripped layer further downstream.

\section{REFERENCES}

BarNetT, D. O. \& Bentley, H. T. 1974 Statistical bias of individual realization laser velocimeters. Proc. 2nd Int. Workshop in Laser Velocimetry, vol. 1, pp. 428-444.

BatT, R. G. 1975 Some measurements on the effect of tripping the two-dimensional shear layer. A.I.A.A.J. 13, 245-247.

Bradshaw, P. 1966 The effect of initial conditions on the development of a free shear layer. J. Fluid Mech. 26, 225-236.

Browand, F. K. \& Latrao, B. O. 1976 The effect of initial conditions upon the growth of the two-dimensional mixing layer. Bull. Am. Phys. Soc. 21, 1221.

Brown, G. L. \& Roshro, A. 1971 The effect of density difference on the turbulent mixing layer. Turbulent Shear Flows. AGARD Conf. Proc. 93, 23-1-23-11.

Brown, G. L. \& Roshiko, A. 1974 On density effects and large structure in turbulent mixing layers. J. Fluid Mech. 64, 775-816.

Dimotakis, P. E. 1976 Single scattering particle laser-Doppler measurements of turbulence. $A G A R D$ Symp. Non-intrusive Instrumentation in Fluid Flow Res., SaintLouis, France, paper 10. 
Dimotakis, P. E. \& Lang, D. B. 1974 Single seattering particle laser-Doppler velocimetry. Bull. Am. Phys. Soc. no. 1145.

LIFPMANN, H. W. \& LAUFER, J. 1947 Investigations of free turbulent mixing. N.A.C.A. Tech. Note, no. 1257.

MCLAUGHIIN, D. K. \& TIEDERMAN, W. G. 1973 Biasing correction for individual realization of laser anemometer measurements in turbulent flows. Phys. Fluids, 16, 20822088.

Mayo, W. T. 1970 Spatial filtering properties of the reference beam in an optical heterodyne receiver. Appl. Optics, 9, 1159-1162.

MOORE, D. W. \& SATFMAN, P. G. 1975 The density of organized vortices in a turbulent mixing layer. J. Fluid Mech. 69, 465-473.

OsTER, D. 1976 An experimental investigation of the two-dimensional turbulent mixing layer. M.Sc. thesis, Tel-Aviv University.

OWEN, P. R. 1970 The decay of a turbulent trailing vortex. Aero. Quart. 61, 69-78.

PHILlIPs, O. M. 1955 The irrotational motion outside a free turbulent boundary. Proc. Camb. Phil. Soc. 51, 220-229.

Roshro, A. 1967 Transition in incompressible near-wakes. Phys. Fluids Suppl. 10, S181-S183.

RosHro, A. 1974. Progress and problems in understanding turbulent shear flows. SQUID Workshop on Turbulent Mixing, Purdue University.

SARohta, V. 1976 Experimental investigation of oscillations in flows over shallow cavities. A.I.A.A. Paper, no. 76-182.

SAto, H. 1956 Experimental investigation on the transition of laminar separated layer. J. Phys. Soc. Japan, 11, 702-709, 1128.

Stegman, A. E. 1966 The antenna properties of optical heterodyne receivers. Appl. Optics, 5, 1588-1594.

SPENCER, B. W. \& JONES, B. G. 1971 Statistical investigation of pressure and velocity fields in the turbulent two stream mixing layer. A.I.A.A. Paper, no. 71-613.

Winant, C. D. 1972 Vortex pairing in a turbulent shear layer at moderate Reynolds numbers. Ph.D. thesis, Dept. of Aerospace Engineering, University of Southern California, Los Angeles.

WINANT, C. D. \& BrowaNd, F. K. 1974 Vortex pairing: the mechanism of turbulent mixing layer growth at moderate Reynolds number. J. Fluid Mech. 63, 237-255.

WYGNaNski, I. \& FiEdLeR, H. E. 1970 The two-dimensional mixing region. J. Fluid Mech. 41, 327-361. 


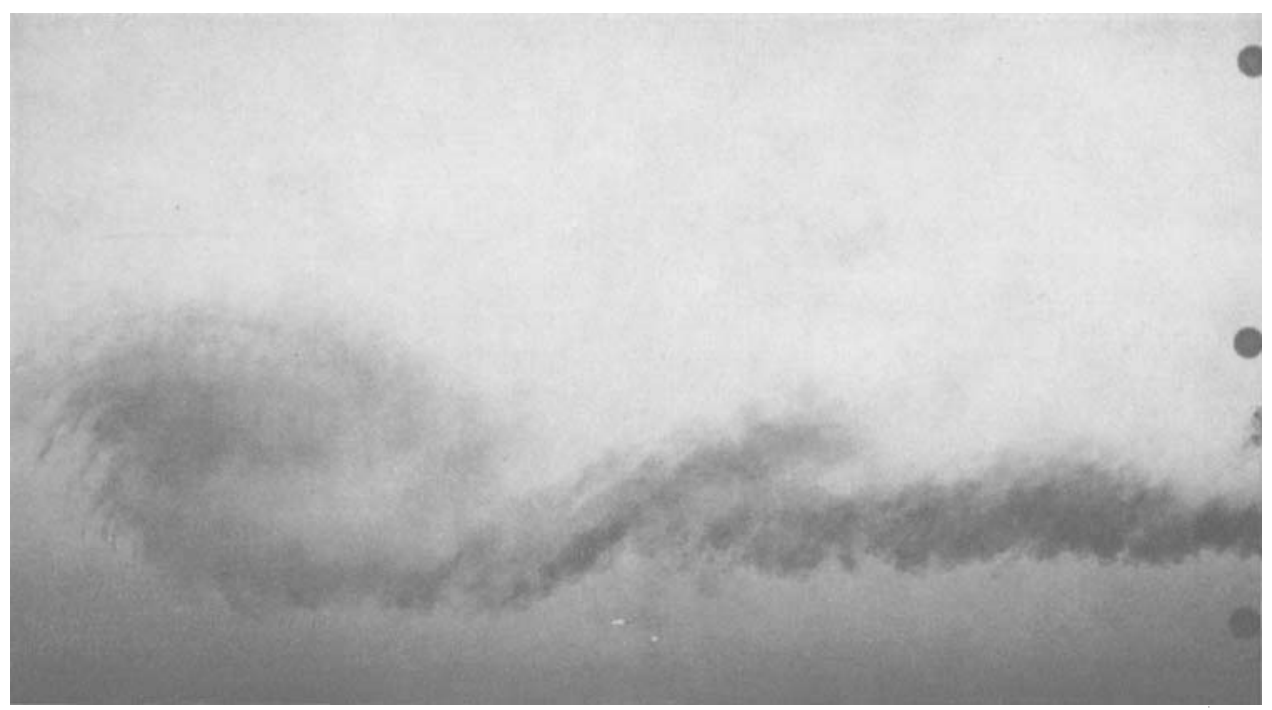

$(a)$

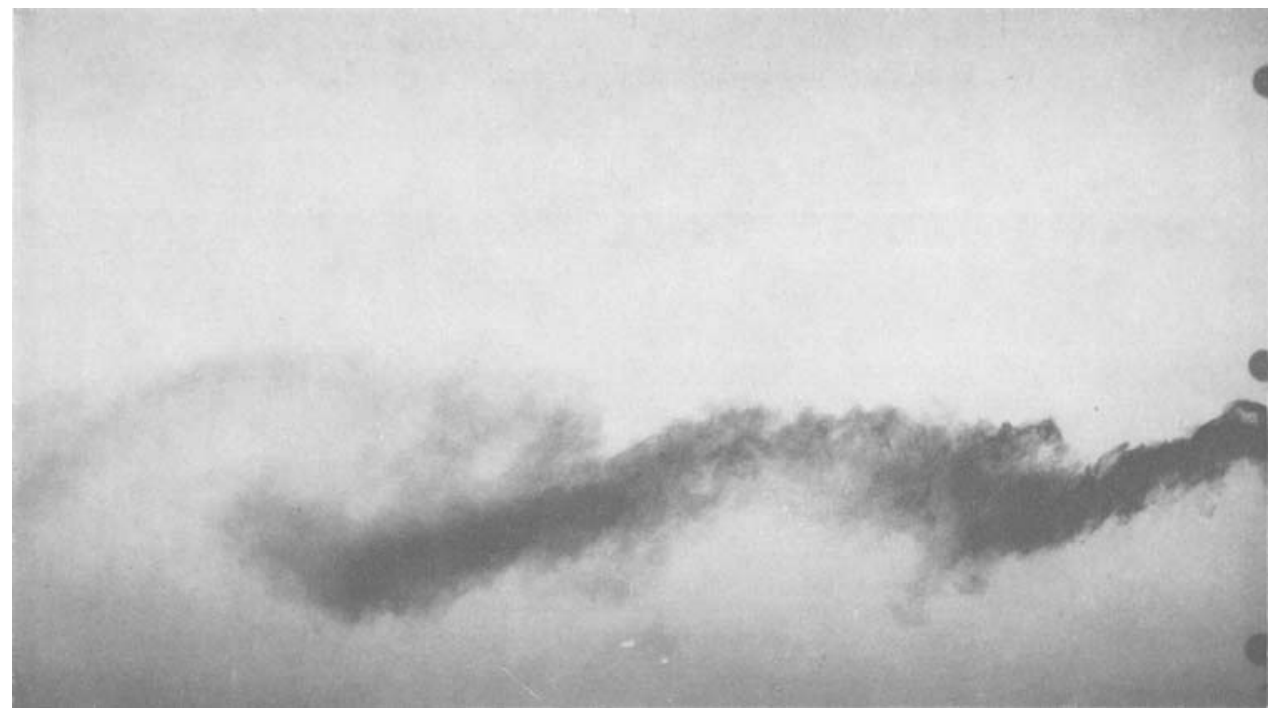

(b)

FIgURe 4. Large structure $120 \mathrm{~cm}$ (picture centre) downstream of the splitter plate; $U_{1}=100 \mathrm{~cm} / \mathrm{s}$, flow from right to left, high-speed side at the bottom. (a) Injection from high-speed side. (b) Injection from low-speed side. 


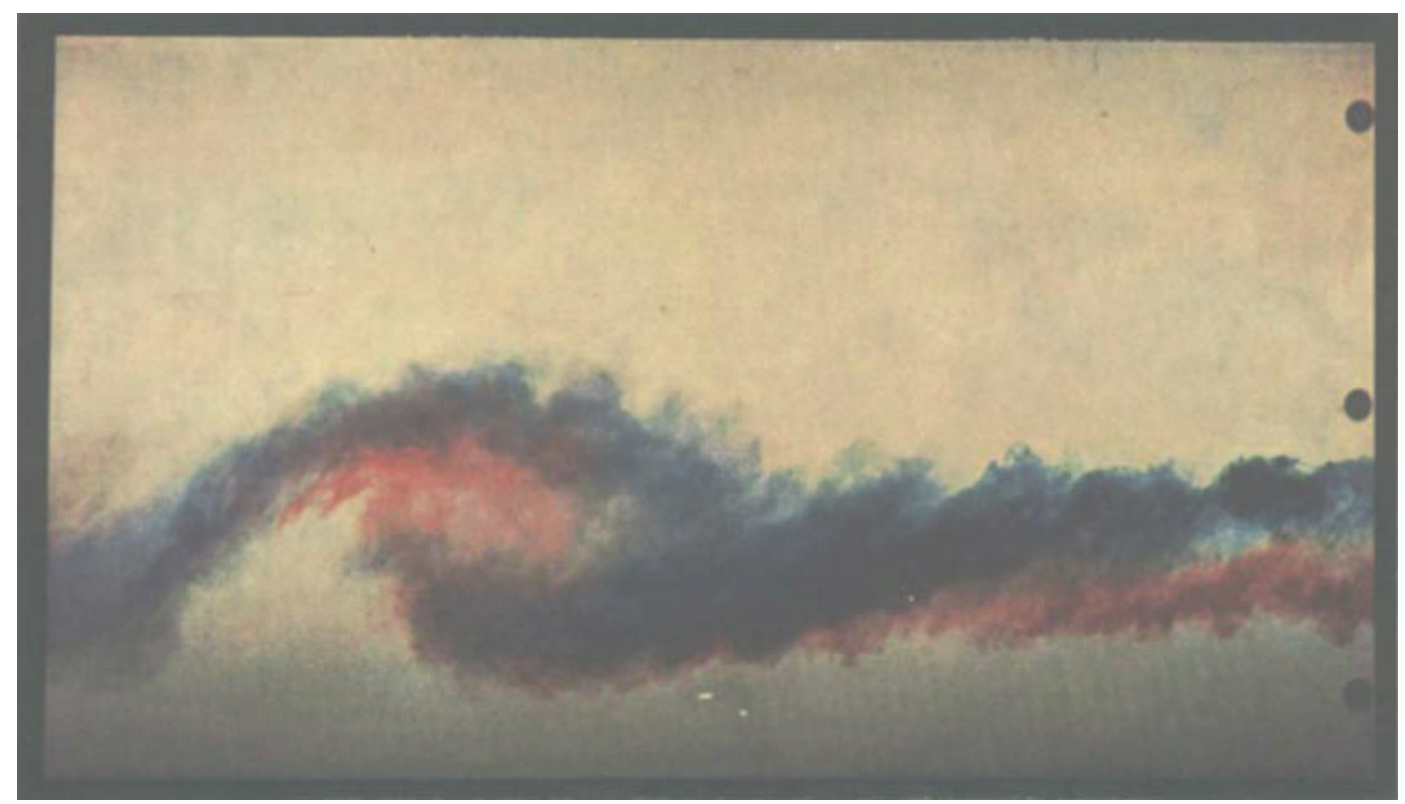

FiguRe 5. Flow as in figure 4. Simultaneous injection from both sides (blue dye from low-speed side, red dye from high-speed side.

Figure 6. Flow as in figure 4. Chemical reaction. 